\title{
Heterologous Expression of the DREB Transcription Factor AhDREB in Populus tomentosa Carrière Confers Tolerance to Salt without Growth Reduction under Greenhouse Conditions
}

\author{
Qi Guo ${ }^{1}$, Nan Lu ${ }^{1}$, Yuhan Sun ${ }^{1}$, Wei Lv ${ }^{1}$, Zijing Luo $^{1}{ }^{1}$, Huaxin Zhang ${ }^{2}$, Qingju Ji ${ }^{3}$, \\ Qingshan Yang ${ }^{4}$, Shouyi Chen ${ }^{5}$, Wanke Zhang ${ }^{5}$ and $\mathrm{Yun} \mathrm{Li}^{1}{ }^{1, *}$ \\ 1 Beijing Advanced Innovation Center for Tree Breeding by Molecular Design, College of Biological Sciences \\ and Technology, National Engineering Laboratory for Tree Breeding, Beijing Forestry University, \\ Beijing 100083, China; Guoqi0529@126.com (Q.G.); ln_890110@163.com (N.L.); syh831008@163.com (Y.S.); \\ 18037935780@163.com (W.L.); luozijingbjfu@163.com (Z.L.) \\ 2 Chinese Academy of Forestry, Beijing 100091, China; 13601283540@126.com \\ 3 Cangzhou Municipal Forestry Seeding and Cutting Management Center, Cangzhou 061001, China; \\ jqj102@163.com \\ 4 Shandong Academy of Forestry, Jinan 250014, China; 15315050868@163.com \\ 5 Institute of Genetics and Developmental Biology, Chinese Academy of Sciences, Beijing 100101, China; \\ sychen@genetics.ac.cn (S.C.); wkzhang@genetics.ac.cn (W.Z.) \\ * Correspondence: yunli@bjfu.edu.cn; Tel./Fax: +86-10-6233-6094
}

Received: 28 January 2019; Accepted: 22 February 2019; Published: 27 February 2019

\begin{abstract}
The DREB transcription factors regulate multiple stress response genes, and are therefore useful for molecular plant breeding. AhDREB, a stress-inducible gene, was isolated from Atriplex hortensis L. and introduced into Populus tomentosa Carrière under the control of the CaMV35S promoter. Under salt stress, the chlorophyll content and net photosynthetic rate were higher in transgenic lines than in the wild type (WT). Moreover, the rate of electrolyte penetration (REC) was lower in the transgenic lines. Additional analyses revealed that the AhDREB transgenic plants generally displayed lower malondialdehyde (MDA) activity but higher superoxide dismutase (SOD) and peroxidase (POD) activities and proline content than the WT under salt stress. RNA sequencing indicated that $A h D R E B$ could enhance tolerance to salt by activating various downstream genes in the transgenic plants. Furthermore, no growth inhibition was detected in transgenic plants expressing AhDREB driven by the constitutive CaMV35S promoter. The transcriptome showed 165 and 52 differentially expressed genes in transgenic plants under stress and non-stress conditions, respectively, among which no significant metabolic pathway was enriched and no unintended effects have yet been identified. Together, these results suggest that $A h D R E B$ may be a good candidate gene for increasing salt tolerance in transgenic poplar breeding.
\end{abstract}

Keywords: Populus tomentosa; AhDREB; salt; unintended effects; photosynthesis; antioxidant defense system

\section{Introduction}

Populus tomentosa Carr. (P. tomentosa) is native to China, where it is mainly distributed in 10 provinces in the northern part of the country. P. tomentosa exhibits many desirable characteristics, such as broad adaptability, a short rotation time, and rapid growth, which make it an important pulp material and afforestation tree species [1]. Given that $P$. tomentosa does not grow well on saline soils, which cover a large area of China, the application and distribution of $P$. tomentosa are seriously 
restricted [2]. High salinity can cause membrane disorganization, decreased photosynthetic activity, metabolic toxicity, generation of reactive oxygen species (ROS), altered nutrient acquisition, and inactivation of enzymes, thereby affecting cell viability and resulting in defective plant growth and even death, which negatively affect the production of crops and woodlands [3-5].

Plants respond and adapt to salt stresses through numerous biochemical reactions and physiological processes that are controlled by many genes. A number of genes and their products have been reported that respond to salt at the transcriptional and translational levels. These proteins can be classified into two groups. One group includes proteins that directly protect against stresses, such as late embryogenesis abundant (LEA) proteins, peroxidase (POD), molecular chaperones, and sodium-hydrogen antiporter (NHX) protein [6-11]. Genes in the other group regulate signal transduction and gene expression during the stress response, including various transcription factors (TFs), protein kinases and other signaling molecules [12]. TFs play a central role in activating the expression of defensive genes and inducing expression of downstream stress-related genes to combat abiotic stresses. One type of TF, DREB proteins of the ERF subfamily, can improve salt stress tolerance in plants. These proteins function through interaction with a dehydration-responsive cis-element found in the core A/GCCGAC sequence of the promoter region of several genes that are induced in response to salt and other abiotic stresses [13]. Due to their ability to regulate a large number of downstream stress-responsive genes, these genes have the potential for improving stress tolerance in transgenic plant breeding [14-16]. Improving the salinity tolerance of the plants through overexpression of DREBs has been accomplished in Arabidopsis, tobacco, rice, wheat, potato, soybean and many other plants. Increasing evidence has shown that DREB proteins play crucial roles in regulating salt stress responses in plants [17-24].

In a previous study, our lab successfully transferred an $A h D R E B$ gene cloned from Atriplex hortensis into a hybrid of $P$. tomentosa through Agrobacterium-mediated transformation. The overexpressed $A h D R E B$ gene significantly improved the survival rate of $P$. tomentosa in salty soil. However, the mechanism by which $A$ hDREB improved the salt tolerance of $P$. tomentosa remains unclear. In addition, tolerance to abiotic stresses is complicated due to the large number of genes and pathways that may be involved [25-27]. Furthermore, the interaction between plants and the environment is an intricate, continuous process that is difficult to characterize, adding to the complexity involved in manipulating abiotic stress tolerance traits. The complexity of these traits may also increase the likelihood of unintended effects arising from overexpressing a TF in transgenic plants [28]. In transgenic systems, two types of unintended effects are known: position effects attributed to the insertion of a foreign gene at a particular locus in the genome and resulting interference, and pleiotropic effects that are independent of the site of transgene insertion and represent the synthesis of phenotypic effects caused by expression of the transgene [29]. Position effects will vary with the site of insertion and can be easily eliminated by selecting transgenic lines with minor or no position effects. However, aside from the intended traits of pleiotropic effects, other pleiotropic effects may occur through unexpected interactions of the genes with plant processes, and therefore constitute unintended effects. These effects are more difficult to eliminate and are more likely to negatively influence the growth of the receptor plant [30]. For this reason, unintended effects must be studied when a foreign gene is used in transgenic breeding.

In this study, our main objective is to demonstrate that ectopic expression of $A h D R E B$ leads to improved salt tolerance in poplar. Such improvement was associated with maintenance of photosynthesis, the antioxidant defense system and osmotic adjustment. RNA sequencing (RNA-seq) analysis indicated that this may be caused by activation of downstream genes involved in cell protection against the adverse effects of salt. Furthermore, there were no negative effects on plant growth when $A h D R E B$ was driven by the strong constitutive CaMV35S promoter, and MapMan analysis of differentially expressed genes between transgenic and non-transgenic poplars showed no significant enrichment of metabolic pathways. 


\section{Materials and Methods}

\subsection{Plant Material and Salt Treatments}

A vector with a T-DNA region containing the nptII gene, which confers resistance to the antibiotic kanamycin, as a selection gene and the AhDREB1 gene was driven by the CaMV35S constitutive promoter and transformed into hybrid Populus $(($ Populus tomentosa $\times$ Populus bolleana L. $) \times$ P. tomentosa $)$ using Agrobacterium tumefaciens Smith \& Townsend in 2003. We selected two transgenic lines, T46 and T12, for our study. The non-transgenic poplar 401, which is also the recipient plant of $A h D R E B 1$, was selected as a control.

Salt treatment experiments were conducted with the two transgenic lines T46 and T12 and the non-transgenic line 401 in a greenhouse with a temperature regime of $25^{\circ} \mathrm{C}$ day $/ 18{ }^{\circ} \mathrm{C}$ night, a $14-\mathrm{h}$ light/10 h-dark photocycle, relative humidity of 70\% day /80\% night, and at a photon flux density of approximately $300-400 \mu \mathrm{mol} \mathrm{m}^{-2} \mathrm{~s}^{-1}$ at Beijing Forestry University in July 2015. In vitro rooted plants were acclimatized in the greenhouse for 2 weeks and then transplanted into plastic pots $(15.0 \mathrm{~cm}$ in diameter and $13.0 \mathrm{~cm}$ tall) containing $2 \mathrm{~kg}$ soil (vermiculite, sand and pearl stone mixed at a ratio of 1:1:1) for several weeks. All pots were placed on plastic plates, saturated with water, and left to drain every 2 days. All plants had water withheld for 3 days before salt treatment.

Salt treatments were initiated when seedlings were approximately $15-20 \mathrm{~cm}$ in height in late August 2015. Randomly selected plants with similar heights, numbers of leaves, and leaf areas were subjected to treatments. To determine the appropriate sodium chloride $(\mathrm{NaCl})$ concentration, five $\mathrm{NaCl}$ concentrations, i.e., 0 (control), $0.2,0.4,0.6$, and $0.8 \%$ (relative to the dry weight of potting soil), were applied to the soils. To achieve these levels of soil salinity, $\mathrm{NaCl}$ was dissolved in $500 \mathrm{~mL}$ distilled water at rates of $0,4,8,12$ and $18 \mathrm{~g}$ and added to the pots to reach water saturation. Then, a plastic pad was laid under each pot to avoid salt drainage during the process. The plants were watered every 2 days to maintain soil moisture at the maximum soil moisture capacity throughout salt treatment. The soil salinity was tested using an EM-38 conductivity meter (Geonics Ltd., Mississauga, ON, Canada) on days 2 and 60 after salt treatment. The soil salinity values are shown in Supplementary Table S1. Three individual plants from each line were used in the experiments, each of which was repeated three times.

\subsection{Determination of the Increment of Growth Parameters}

Before and 60 days after salt treatment, plant length and base diameter were determined. The height of plants was measured using a ruler, and base diameters was determined using a caliper. The increments of plant height and base diameter were calculated using the following formula:

Plant height increment

$=$ plant height after 15 days of salt treatment

- plant height after 0 days of salt treatment

Base diameter increment

$=$ base diameter after 15 days of salt treatment

-base diameters after 0 days of salt treatment

\subsection{Determination of Electrolyte Leakage and Malondialdehyde (MDA) Concentration}

Electrolyte leakage of non-transgenic and transgenic plants was determined on day 60 according to the method of Wang et al. [15]. In short, leaves were thoroughly rinsed with deionized water, and then 5-g round sections were cut from the leaves of each sample and placed into a clean beaker with $30 \mathrm{~mL}$ of deionized water under vacuum for $15 \mathrm{~min}$. The electrical conductivity was measured and denoted $\mathrm{r} 1$. The leaves were then heated at $90{ }^{\circ} \mathrm{C}$ for $20 \mathrm{~min}$ and cooled at room temperature (about $25^{\circ} \mathrm{C}$ ). Electrical conductivity was measured again, and this value was labeled $\mathrm{r} 2$. The formula for calculation of electrolyte leakage was (r1/r2) 100\%. 
The MDA concentration was measured on day 60 according to the method of Quan et al. (2004) [31] using a thiobarbituric acid (TBA) colorimetric assay, in which $0.5 \mathrm{~g}$ of fresh tissue was homogenized in $2 \mathrm{~mL} 10 \%$ trichloroacetic acid (TCA, $w / v)$. The MDA concentration was defined as

$$
\mathrm{C}=6.45 \times(\mathrm{OD} 532-\mathrm{OD} 600)-0.56 \times \mathrm{OD} 450
$$

\subsection{Measurement of Leaf Gas Exchange}

Leaf gas exchange parameters, including net assimilation rate $\left(\mathrm{Pn}, \mu \mathrm{mol} \mathrm{m}{ }^{-2} \mathrm{~s}^{-1}\right)$, stomatal conductance $\left(\mathrm{Gs}, \mathrm{mol} \mathrm{m} \mathrm{m}^{-2} \mathrm{~s}^{-1}\right)$, transpiration rate $\left(\mathrm{Tr}, \mathrm{mmol} \mathrm{m} \mathrm{m}^{-2} \mathrm{~s}^{-1}\right)$, and intercellular $\mathrm{CO}_{2}$ concentration $\left(\mathrm{Ci}, \mu \mathrm{mol} \mathrm{mol}{ }^{-1)}\right.$, were measured on the fourth fully expanded top leaves of three plants (bout the 4th to 6th leaves below the apex, and we have re-written this part) per treatment on day 60 using an internal light source with a photosynthetically active radiation (PAR) value of $1000 \mu \mathrm{mol} \mathrm{m}{ }^{-2} \mathrm{~s}^{-1}$. During measurement, the ambient air $\mathrm{CO}_{2}$ concentration was approximately $400 \mu \mathrm{mol} \mathrm{mol}{ }^{-1}$ and the temperature was approximately $25^{\circ} \mathrm{C}$. All measurements were performed in triplicate.

\subsection{Measurement of Proline, SOD, POD and Chl Content}

The proline and antioxidant enzyme activities were detected on day 60 . The second or third fully expanded top leaves were collected and frozen in liquid nitrogen, then stored at $-80^{\circ} \mathrm{C}$.

Proline was extracted and quantitated using the method of Bates et al. (1973) [32]. Briefly, $0.25 \mathrm{~g}$ samples were homogenized with $2 \mathrm{~mL} \mathrm{3 \%}$ sulfosalicylic acid and the homogenate was centrifuged at $4000 \mathrm{rpm}$ for $20 \mathrm{~min}$. The supernatant was treated with acetic acid and acid ninhydrin and boiled for $1 \mathrm{~h}$, and the absorbance at $520 \mathrm{~nm}$ was then measured. Proline (Sigma-Aldrich) was used to generate a standard curve.

Fresh leaf samples were obtained and frozen instantly in liquid $\mathrm{N}$ and subsequently stored at $-80^{\circ} \mathrm{C}$. Frozen leaf samples of $0.5 \mathrm{~g}$ were ground to fine powder in liquid $\mathrm{N}$ using a mortar and then homogenized with $3 \mathrm{~mL}$ of $50 \mathrm{mM}$ phosphate buffer solution (pH 7.8) containing $1 \mathrm{mM}$ EDTA-Na ${ }^{2+}$ and $1 \%$ polyvinylpyrrolidone (PVP). The homogenate was centrifuged at 10,000 rpm for $15 \mathrm{~min}$, and the supernatant was then centrifuged at 10,000 rpm for an additional $5 \mathrm{~min}$. The supernatant was collected as a crude enzyme extract for enzyme measurements and stored at $4{ }^{\circ} \mathrm{C}$. All steps were carried out at $4{ }^{\circ} \mathrm{C}$. Enzyme activities were assayed using a spectrophotometer.

The total SOD activity was assayed according to Becana et al. (1986) [33] based on inhibition of the photochemical reduction of nitroblue tetrazolium (NBT). The reaction was initiated by the addition of $100 \mu \mathrm{L}$ of crude enzyme extract. The entire system was positioned $30 \mathrm{~cm}$ below a light source (six $15-\mathrm{W}$ fluorescent tubes) for $30 \mathrm{~min}$. The reaction was stopped by turning off the light. The complete reaction mixture without enzyme extract was incubated under the same light used for the experimental samples to provide a light blank, and the complete reaction mixture including $100 \mu \mathrm{L}$ of enzyme extract was incubated in the dark to provide a dark blank. The reduction in the amount of NBT was determined by monitoring the change in absorbance at $560 \mathrm{~nm}$. The readings obtained from the light blank were used to determine units of enzymatic activity. One unit (U) of SOD enzyme activity was defined as the amount of enzyme that produced $50 \%$ inhibition of NBT reduction under the assay conditions, expressed as U SOD activity $\mathrm{mg}^{-1}$ protein.

The POD activity of enzyme extracts was assayed by monitoring changes in absorbance at $470 \mathrm{~nm}$ in mixtures containing $0.02 \mathrm{M} \mathrm{Na} 2 \mathrm{HPO} 4,0.08 \mathrm{M} \mathrm{NaH}_{2} \mathrm{PO}_{4}, 20 \mathrm{mM}$ guaiacol, $4 \mathrm{mM} \mathrm{H}_{2} \mathrm{O}_{2}$, and enzyme extract (10 mL), pH 6, in a total volume of $3 \mathrm{~mL}$ (Civello 1995) [34].

To measure $\mathrm{Chl}$ variation, non-transgenic and transgenic plants were evaluated on the day of the salt treatment and 60 days later using the portable chlorophyll meter SPAD-502 Plus (Konica Minolta Holdings, Inc. Chiyoda-ku, Tokyo, Japan). The second healthy and fully expanded leaves from the top were measured 10 times on each leaf and the $\mathrm{Chl}$ contents were calculated as the average value. All measurements were performed in triplicate. Chl variation was calculated with the following formula: 


\subsection{Cdna Library Preparation and Illumina Transcriptomic Sequencing}

To further investigate the role of $A h D R E B$ in the mechanism of salt resistance in transgenic P. tomentosa, the non-transgenic line 401 and transgenic lines T-46 and T-12 treated with $0 \%$ and $0.6 \% \mathrm{NaCl}$ were subjected to transcriptomic analysis following salt treatment and the sequencing data has submitted to NCBI database (accession number: PRJNA522057). Total RNA from the mixed sample (three individual plants) was isolated using the RNA EasySpin Isolation System (Aidlab Biotech, Beijing, China) according to the manufacturer's protocol. Following treatment with RNase-free DNase I (New England BioLabs) for $30 \mathrm{~min}$ at $37^{\circ} \mathrm{C}$ to remove residual DNA, sequencing was performed by Shanghai Biotechnology Co. Ltd., where a cDNA library was constructed and subjected to Illumina HiSeq2500 sequencing, as described by Sun et al. [35].

\subsection{Analysis of Illumina Transcriptomic Sequencing Results}

The RNA-seq reads were mapped onto the genome of Populus trichocarpa (JGI v3.0, https: / / phytozome. jgi.doe.gov/pz/\#!linfo?alias=Org_Ptrichocarpa) using TopHat (v.2.0.9, allowing two mismatches per read as the default).

\subsection{Differential Expression Analysis and Function Enrichment}

Gene expression levels were estimated as reads per kilobase of exon region in a given gene per million mapped reads (RPKM) [36]. Two parameters were used to identify differentially expressed transcripts (DETs) between the transgenic and non-transgenic samples: a fold change of not less than 2 (an absolute value of $\log 2$ Ratio (T-46 (or T-12)/401) $\geq 1$ ) and a false discovery rate (FDR) adjustment with a significance level of 0.05 . Gene Ontology (GO) and pathway enrichment analyses were then performed with a cutoff $P$ value of 0.05 compared to the whole transcriptome as background. To study the mechanism through which $A h D R E B$ improved salt tolerance, we screened differentially expressed genes between T-46 and 401, as well as T-12 and 401 , under $0.6 \% \mathrm{NaCl}$ salt treatment. To investigate potential unintended pleiotropic effects in AhDREB transgenic poplar, we screened differentially expressed genes between T-46 and 401, as well as T-12 and 401 under $0 \% \mathrm{NaCl}$ salt treatment. In addition, to minimizing transgene position effects, only genes that were significantly affected in both T-46 and T-12 were treated as differentially expressed.

The MapMan-based functional categorization of all differentially expressed genes was performed by comparing their protein sequence to that of Arabidopsis TAIR10 (http://www.arabidopsis.org/) using the standalone version of NCBI BLASTP (2.2.31+) with the default settings. MapMan categorization was transferred from TAIR10. To evaluate whether up-regulated genes in transgenic plants associated with salt resistance were directly regulated by $A h D R E B, 2000$-bp sequences upstream of the $5^{\prime}$ UTR region (regarded as the promoter region) were downloaded (JGI v3.0, https: / / phytozome. jgi.doe.gov /pz/\#! info?alias=Org_Ptrichocarpa) and the presence of the cis-regulatory element CCGAC was detected [37].

\section{Results}

\subsection{Growth under Increasing Levels of Soil Salinity}

Saline conditions had negative effects on plant growth, leading to its reduction. The plant height increments of transgenic and non-transgenic lines showed no significant difference (Figure 1), but as the salt concentration increased, the plant height increment of transgenic lines was significantly higher than that of non-transgenic lines. For treatments of under $8 \%$, reduction in the growth of non-transgenic plants was observed. The base diameter increments were not significantly different between transgenic and non-transgenic lines, except in the 6\%o treatment (Figure 2). 


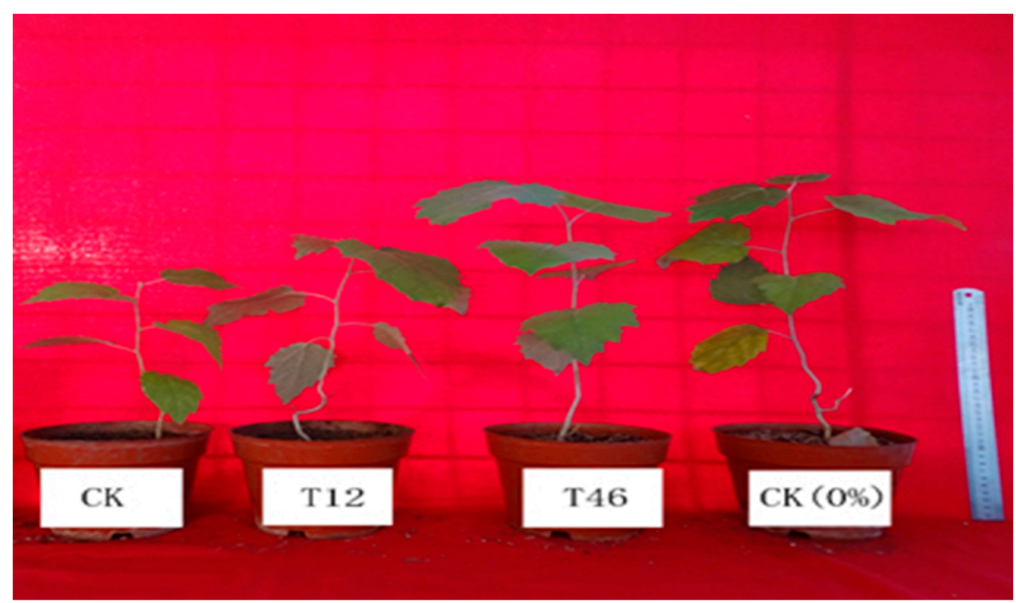

Figure 1. Growth after sodium chloride $(\mathrm{NaCl})$ treatment. CK: non-transgenic poplar treated with $0.6 \%$ $\mathrm{NaCl}$ after 15 days, T-12: transgenic line T-12 treated with $0.6 \% \mathrm{NaCl}$ after 15 days; $\mathrm{T}-46$ : transgenic line $\mathrm{T}-46$ treated with $0.6 \% \mathrm{NaCl}$ after 15 days, $\mathrm{CK}(0 \%)$ : non-transgenic poplar treated with $0 \% \mathrm{NaCl}$ after 15 days.
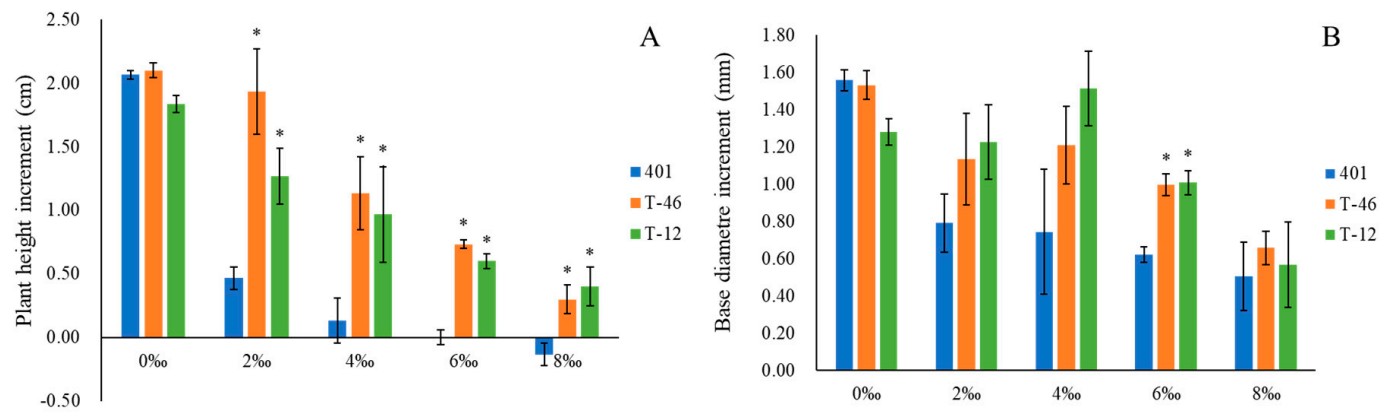

Figure 2. The stem height (A) and base diameter (B) increases of poplar after $\mathrm{NaCl}$ treatment. "*" denotes a significant difference $(p<0.05, \alpha=0.05)$ between treatments. Error bars represent the standard deviation.

\subsection{Effect of Ahdreb Expression on Rate of Electrolyte Penetration (REC) and MDA Contents}

The REC and MDA contents were determined after 60 days of salt treatment. Compared to non-transgenic line 401, the two transgenic lines showed significantly lower REC and MDA contents, especially with high salt treatment (8\%); the REC and MDA contents of the non-transgenic line were $72.31 \%$ and $8.37 \mathrm{nmol} / \mathrm{mg}(\mathrm{FW})$, about 2.6-fold and 2.0-fold higher than that of T-12, respectively (Figure 3A,B).

\subsection{Effect of Ahdreb Expression on Chl Content Variation, SOD and POD Activities And Proline Content}

The variations in proline content of transgenic lines and non-transgenic lines were similar to those of SOD. Pro contents increased with the salt concentration, reaching their peak at $0.6 \%$, and then decreased when treated with $0.8 \% \mathrm{NaCl}$ solution. The transgenic lines had significantly higher Pro contents than the non-transgenic line (Figure 3C).

Salt stress responses were further analyzed by monitoring the activities of superoxide dismutase (SOD) and POD, which scavenge harmful ROS that accumulate during stress. The overall variation in the activities of the two antioxidant enzymes following 60 days of salt stress was similar between the two transgenic lines, while the SOD activity of the transgenic lines was significantly higher than that of the non-transgenic line under all salt treatments (Figure 3D). The POD activity of transgenic lines was significantly higher than that of non-transgenic lines under the $0 \%, 2 \%, 4 \%$ and $8 \% 0 \mathrm{NaCl}$ treatments (Figure 3E). 
Chl content was detected after 0 and 60 days of salt stress. The Chl content decreased with increasing $\mathrm{NaCl}$ concentration in soil, and varied significantly more widely in non-transgenic plants compared to transgenic lines, especially at higher salt levels (6 and 8\%). Transgenic lines were only significantly higher than the non-transgenic line for the $6 \%$ salt treatment (Figure 3F).
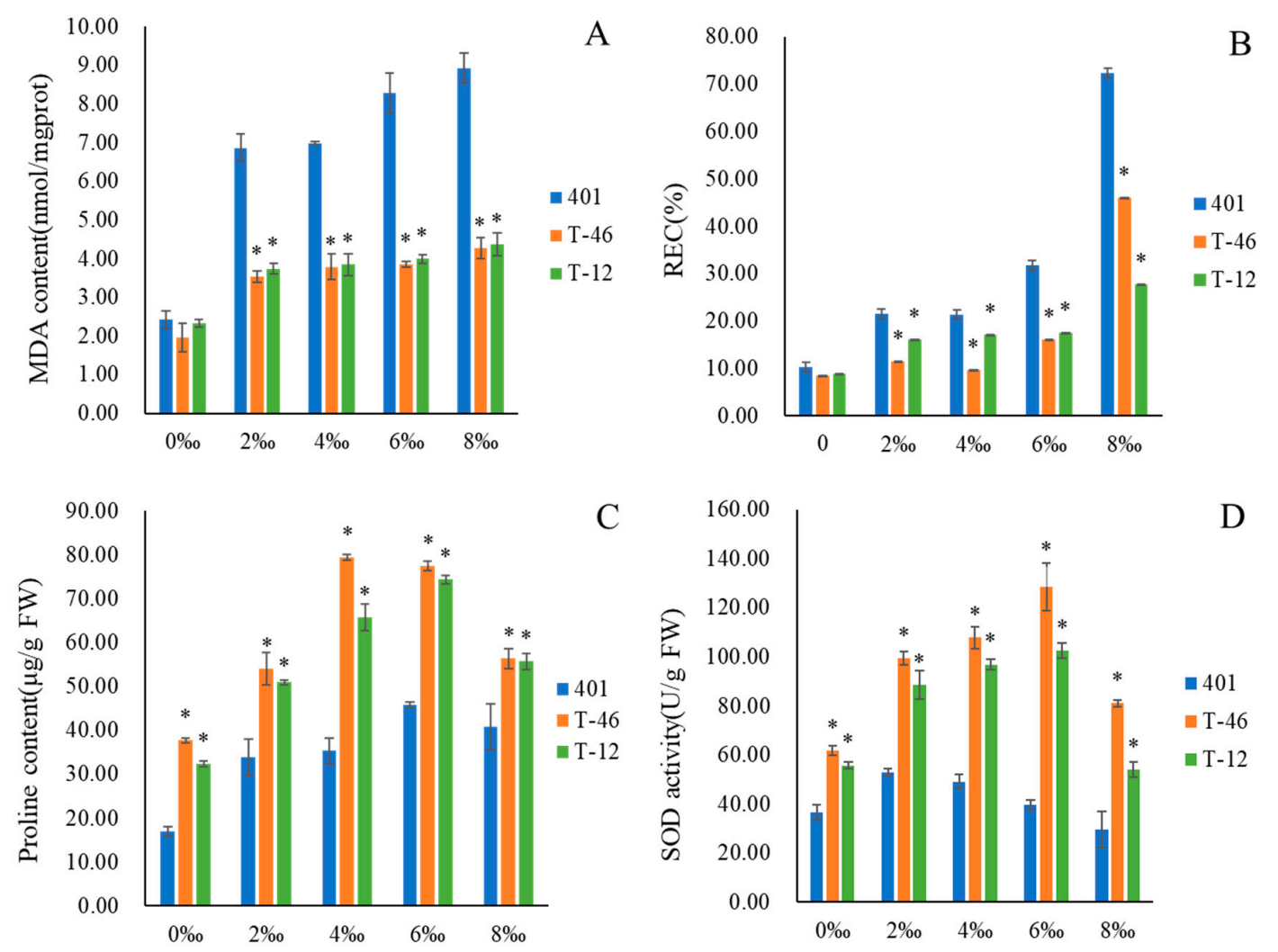

$\mathrm{C}$
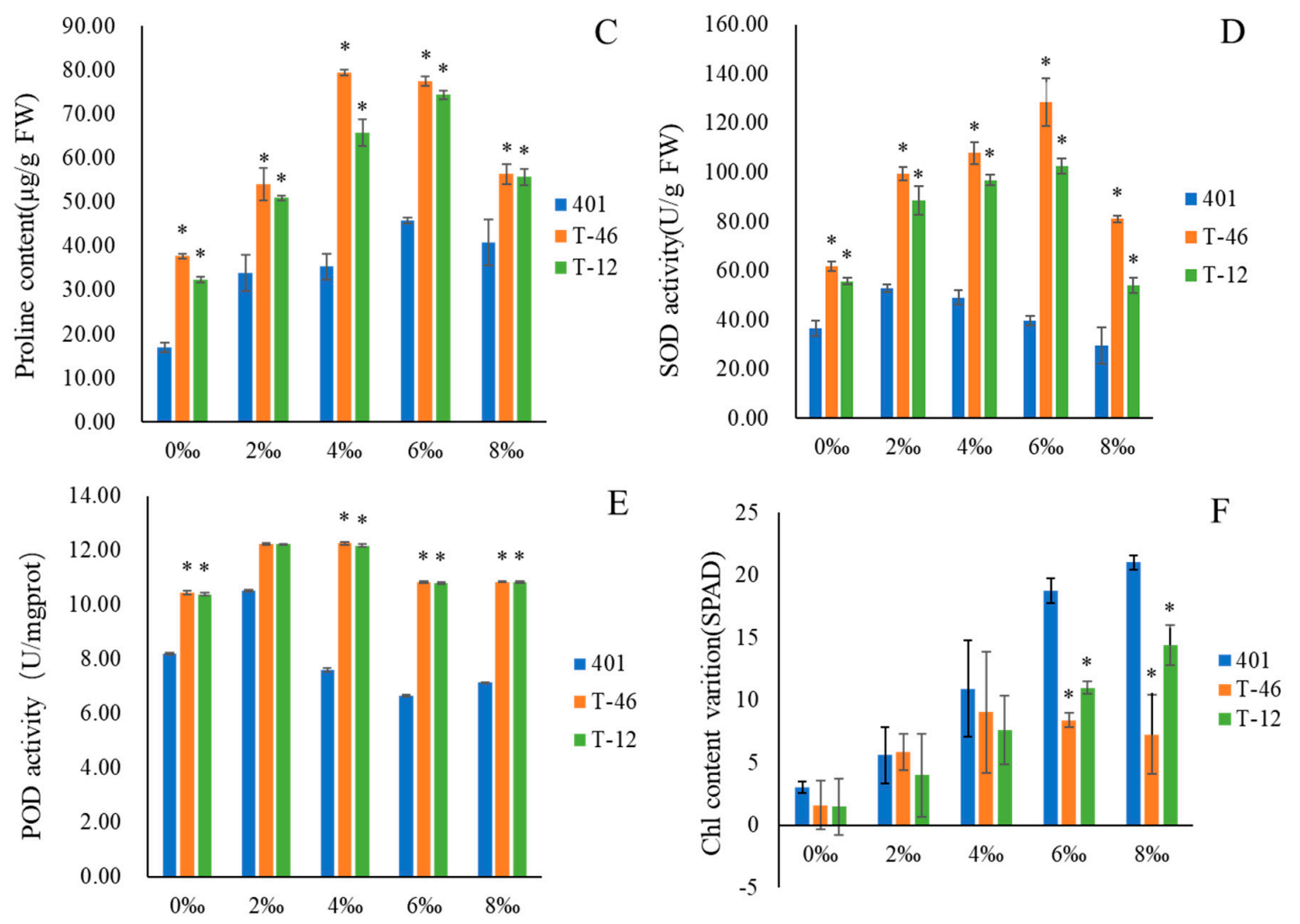

Figure 3. The variations in physiological and biochemical properties after $\mathrm{NaCl}$ treatment: Malondialdehyde (MDA) content (A), relative electrolyte leakage (B), proline content (C), SOD activity (D), POD activity (E), chlorophyll content (F). “*” denotes a significant difference $(p<0.05, \alpha=0.05)$ between treatments. Error bars represent the standard deviation.

\subsection{Photosynthetic Capacity of Transgenic Plants Under Salt Stress Conditions}

To test photosynthetic capacity, we measured net photosynthesis (Pn), Tr and Gs of non-transgenic and transgenic plants. Under non-stress conditions, plants of different lines showed little variability. However, when subjected to salt, non-transgenic line 401 exhibited a significant decrease in Pn, Gs and $\mathrm{Tr}$, while transgenic lines T46 and T12 were less affected (Figure 4). 

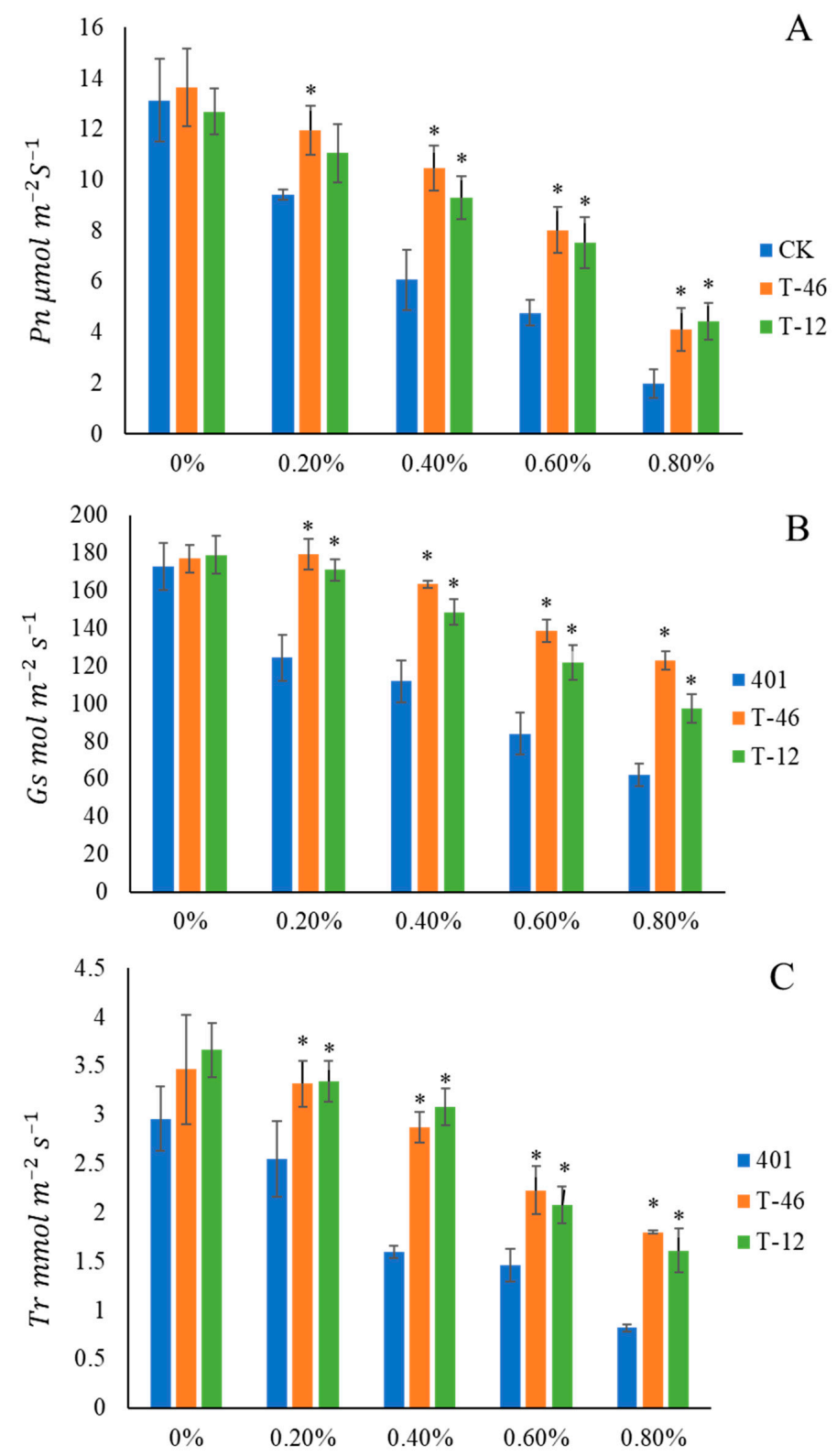

Figure 4. Variations in photosynthesis parameters after $\mathrm{NaCl}$ treatment, net photosynthesis rate, $\mathrm{Pn}(\mathrm{A})$; stomatal conductance, Gs (B); transpiration rate, $\operatorname{Tr}(\mathbf{C}) ;$ “*” denotes a significant difference $(p<0.05$, $\alpha=0.05)$ between treatments. Error bars represent the standard deviation.

\subsection{Transcriptomic Analysis of Ahdreb-Overexpressing Populus Tomentosa Under Salt Stress}

To further investigate the molecular mechanisms involved in the observed enhancement of salt tolerance in $A h D R E B$-overexpressing lines, global expression profiling was conducted to compare transgenic lines T-46 and T-12 and non-transgenic line 401 (wild types; WT) plants following $0.6 \% \mathrm{NaCl}$ treatment. Mixed RNA samples from three separate 120-day-old plants (after 60 days of salt treatment) were used for high-throughput sequencing and in total, 47,800,427 (401, 0\%), 46,925,138 (401, 0.6\%), $43,434,145$ (T46, 0\%), 50,561,810 (T46, 0.6\%), 45,666,581 (T12, 0\%) and 64,667,540 (T12, 0.6\%) raw reads were identified, with 20,628,629 (401, 0\%), 20,668,223 (401, 0.6\%), 18,713,319 (T46, 0\%), 21,591,536 (T46, $0.6 \%), 19,276,874$ (T12, 0\%) and 28,171,096 (T12, 0.6\%) unique reads mapped (Supplemental Table S2).

To identify genes that may play important roles in improving salt tolerance, we focused on 231 differentially expressed genes (165 up-regulated and 66 down-regulated) in the two transgenic lines following salt treatment. Genes that were only differentially expressed in either T46 or T12 were 
excluded as effects caused by foreign gene insert position. A total of 46 genes related to growth, wood, and reproduction genes were identified, including 22 growth-related genes (6 down-regulated, 16 up-regulated), 12 reproduction-related genes (8 up-regulated, 4 down-regulated), and 12 genes correlated with mortality (10 up-regulated, 2 down-regulated) (Supplemental Table S3).

Among the 165 up-regulated genes, we found a total of 51 genes that may be associated with stress resistance, including 10 TFs (3 WRKY, 1 NAC, 3 MYB and 3 BHLH) and 41 functional genes (Table 1). The cis-regulatory element CCGAC was found in 36 of the 51 genes. MapMan functional categorization showed that most differentially expressed genes participated in the metabolic pathways of "ascorbic acid metabolism", "glutathione metabolism" and "flavonoid metabolism", but we did not find that differentially expressed genes were significantly enriched in a specific metabolic pathway (Figure 5).

Table 1. Genes associated with stress resistance among up-regulated genes in transgenic poplar.

\begin{tabular}{|c|c|c|c|c|}
\hline Gene ID & Function Description & Tair ID & $\log 2 \mathrm{FC}$ & $p$-Value \\
\hline \multicolumn{5}{|c|}{ Genes have cri-regulatory element "CCGAC" in promotor regions } \\
\hline Potri.002G186600 & WRKY transcription factor & AT4G01720.1 & Inf & $2.42 \times 10^{-4}$ \\
\hline Potri.004G002800 & Phosphotyrosine protein phosphatases superfamily protein & AT1G05000.1 & Inf & $1.27 \times 10^{-3}$ \\
\hline Potri.011G021100 & Phosphotyrosine protein phosphatases superfamily protein & AT4G03960.1 & 1.22 & $1.54 \times 10^{-3}$ \\
\hline Potri.013G083600 & Peroxidase superfamily protein & AT5G05340.1 & 6.23 & $3.52 \times 10^{-45}$ \\
\hline Potri.018G003800 & NAC transcription factor & AT2G24430.2 & 4.27 & $8.08 \times 10^{-5}$ \\
\hline Potri.005G164900 & myb transcription factor & AT1G34670.1 & 4.04 & $4.48 \times 10^{-4}$ \\
\hline Potri.008G073700 & laccase 5 & AT2G40370.1 & 4.95 & $1.75 \times 10^{-7}$ \\
\hline Potri.006G049200 & heat shock transcription factor B3 & AT2G41690.1 & 4.63 & $1.55 \times 10^{-13}$ \\
\hline Potri.002G015100 & glutathione S-transferase F11 & AT3G03190.1 & 1.38 & $2.08 \times 10^{-5}$ \\
\hline Potri.001G105200 & glutathione peroxidase 6 & AT4G11600.1 & 1.07 & $2.23 \times 10^{-3}$ \\
\hline Potri.006G044600 & dehydration-induced protein (ERD15) & AT2G41430.5 & 1.40 & $1.86 \times 10^{-3}$ \\
\hline Potri.007G072100 & cytochrome P450, family 86 , subfamily B, polypeptide 1 & AT5G23190.1 & Inf & $4.64 \times 10^{-3}$ \\
\hline Potri.004G106600 & cytochrome P450, family 82 , subfamily G, polypeptide 1 & AT3G25180.1 & 3.18 & $1.68 \times 10^{-24}$ \\
\hline Potri.001G334700 & cytochrome P450, family 82 , subfamily C, polypeptide 4 & AT4G31940.1 & 1.27 & $6.94 \times 10^{-4}$ \\
\hline Potri.003G146800 & cytochrome P450, family 78 , subfamily A, polypeptide 6 & AT2G46660.1 & 1.50 & $1.31 \times 10^{-4}$ \\
\hline Potri.003G146800 & cytochrome P450, family 78 , subfamily A, polypeptide 6 & AT2G46660.1 & 1.50 & $1.31 \times 10^{-4}$ \\
\hline Potri.014G072300 & cytochrome P450, family 704, subfamily A, polypeptide 2 & AT2G45510.1 & 1.07 & $1.41 \times 10^{-4}$ \\
\hline Potri.001G167800 & Cytochrome P450 superfamily protein & AT5G07990.1 & 2.30 & $2.47 \times 10^{-5}$ \\
\hline Potri.014G019200 & cytochrome B5 isoform D & AT5G48810.1 & 4.41 & $2.60 \times 10^{-5}$ \\
\hline Potri.017G054300 & cytochrome B5 isoform B & AT2G32720.1 & 2.36 & $1.71 \times 10^{-6}$ \\
\hline Potri.004G149100 & cold-regulated 413-plasma membrane 2 & AT3G50830.1 & 2.07 & $7.77 \times 10^{-7}$ \\
\hline Potri.009G116400 & cold, circadian rhythm, and RNA binding 1 & AT4G39260.3 & 1.95 & $2.25 \times 10^{-9}$ \\
\hline Potri.005G179200 & thylakoidal ascorbate peroxidase & AT1G77490.1 & 1.92 & $4.0 \times 10^{-4}$ \\
\hline Potri.012G002500 & pyruvate kinase family protein & AT3G49160.1 & 3.763 & $2.50 \times 10^{-4}$ \\
\hline Potri.007G021300 & S-adenosyl-L-methionine-dependent methyltransferases superfamily protein & AT3G11480.1 & 4.36 & $6.45 \times 10^{-48}$ \\
\hline Potri.006G222200 & S-adenosyl-L-methionine-dependent methyltransferases superfamily protein & AT5G19530.1 & 1.78 & $8.77 \times 10^{-8}$ \\
\hline Potri.001G404600 & Peroxisomal membrane $22 \mathrm{kDa}$ family protein & AT4G21380.1 & 1.47 & $5.56 \times 10^{-8}$ \\
\hline Potri.008G106400 & Peroxidase superfamily protein & AT2G39470.1 & 3.17 & $1.12 \times 10^{-23}$ \\
\hline Potri.005G195700 & Peroxidase superfamily protein & AT3G11430.1 & 3.64 & $5.63 \times 10^{-31}$ \\
\hline Potri.006G221800 & myb transcription factor 4 & AT4G00430.1 & 1.07 & $1.40 \times 10^{-3}$ \\
\hline Potri.002G173900 & myb transcription factor 3 & AT2G43460.1 & 3.60 & $2.47 \times 10^{-24}$ \\
\hline Potri.005G234500 & salt tolerance homolog2 & AT1G75540.1 & 2.08 & $3.02 \times 10^{-9}$ \\
\hline Potri.009G037300 & highly ABA-induced PP2C gene 2 & AT2G37170.1 & 4.04 & $9.20 \times 10^{-9}$ \\
\hline Potri.004G235400 & cytochrome P450, family 707, subfamily A, polypeptide 1 & AT4G18550.1 & 3.07 & $3.43 \times 10^{-7}$ \\
\hline Potri.003G066400 & Cytochrome P450 superfamily protein & AT1G61720.1 & 3.01 & $4.89 \times 10^{-21}$ \\
\hline Potri.001G167900 & Cytochrome P450 superfamily protein & AT2G33510.1 & 2.26 & $2.81 \times 10^{-10}$ \\
\hline Potri.005G113400 & basic helix-loop-helix (bHLH) DNA-binding superfamily protein & AT1G06550.1 & 2.76 & $1.43 \times 10^{-5}$ \\
\hline Potri.009G107600 & 2-oxoglutarate (2OG) and $\mathrm{Fe}(\mathrm{II})$-dependent oxygenase superfamily protein & AT5G13930.1 & 1.95 & $1.06 \times 10^{-8}$ \\
\hline
\end{tabular}




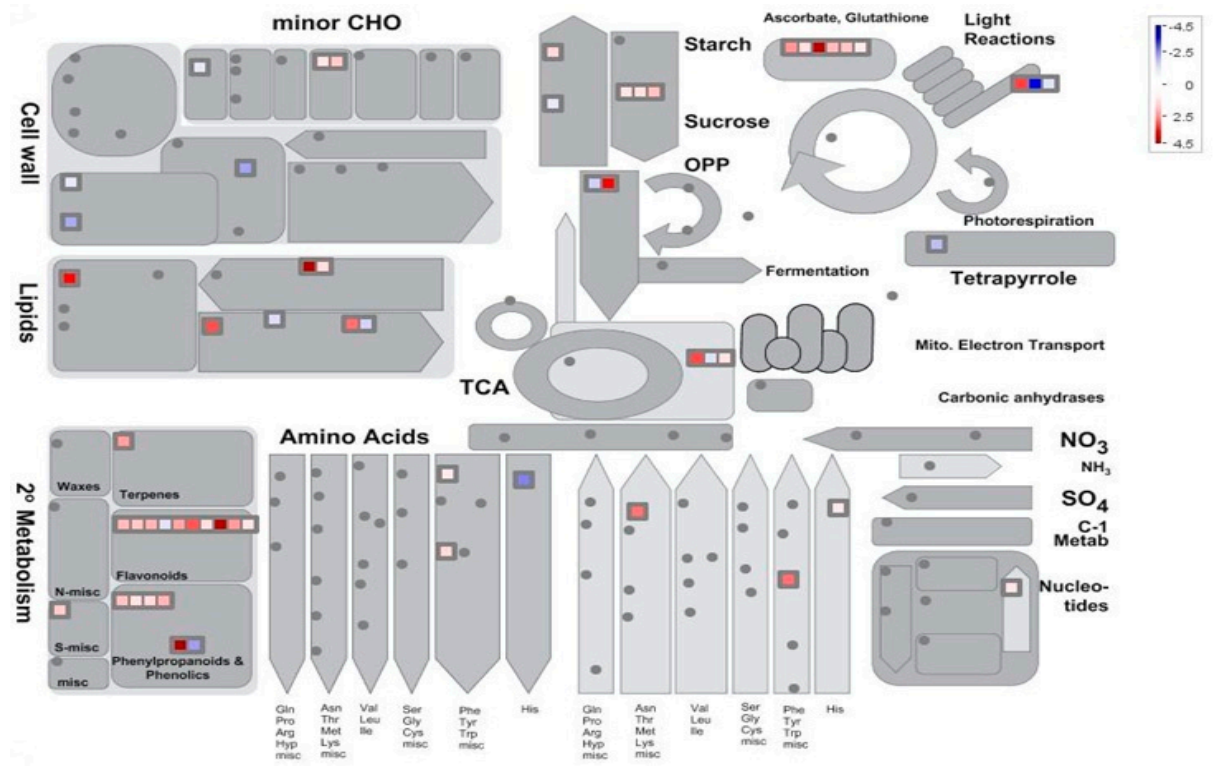

Figure 5. Differentially expressed gene function enrichment analysis after $\mathrm{NaCl}$ treatment.

\subsection{Effect of Ahdreb Overexpression on the Transcriptome in the Absence of Salt}

Transcriptomic analysis of T-46 and T-12 was performed to identify unintended effects resulting from overexpression of the TF AhDREB in the absence of stress. The two independent plant lines containing the $A h D R E B$ transgene were compared to the non-transgenic plant line. The common differentially expressed genes in the two transgenic lines are listed in Table 2. We found 22 total up-regulated and 30 total down-regulated differentially expressed genes. These genes may be influenced by overexpression of the AhDREB gene. In this study, we mainly focus on genes that may influence the growth and wood properties of host plants. Among the 52 differentially expressed genes, 6 were for uncharacterized proteins, 2 were TFs and 44 were functional genes. No metabolic pathway was significantly enriched according to MapMan functional categorization, and we did not find differentially expressed genes significantly enriched in a specific metabolic pathway in MapMan functional categorization (Figure 6).

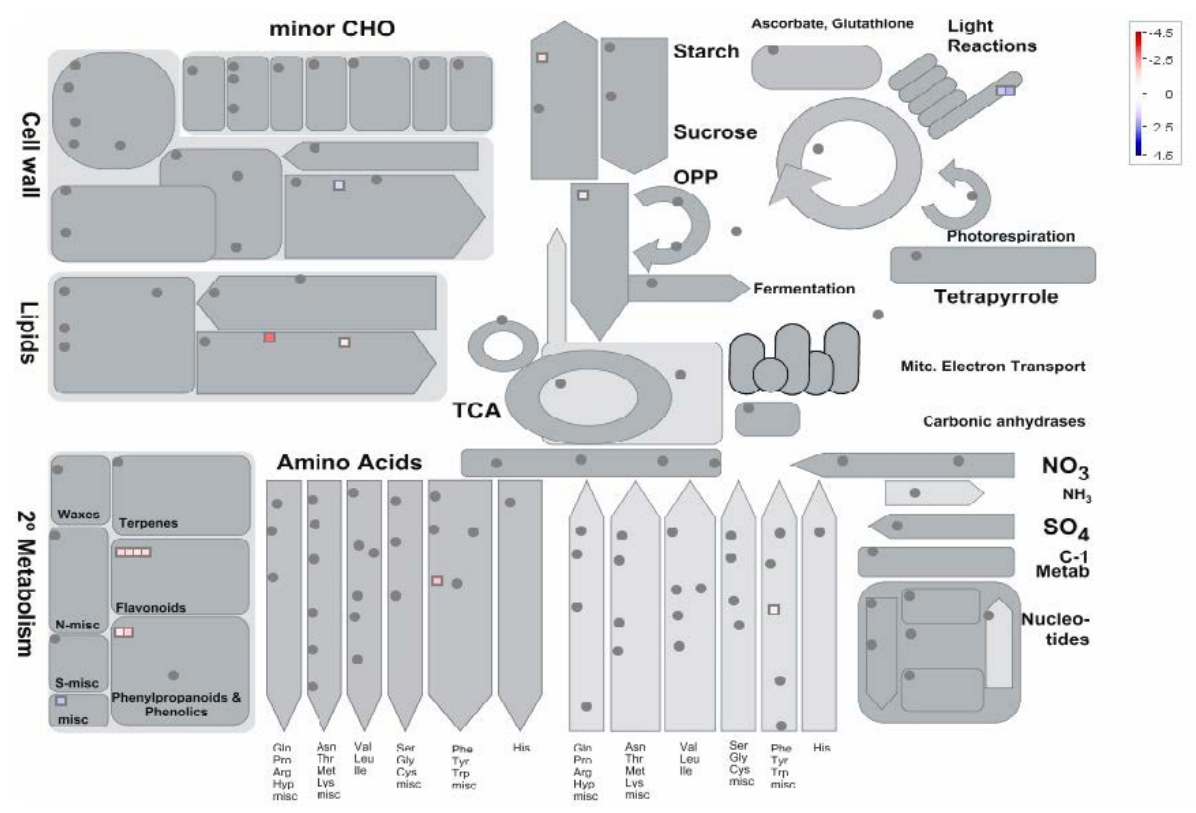

Figure 6. Differentially expressed gene function enrichment analysis under non-stress conditions. 
Table 2. Differentially expressed genes in transgenic poplar under non-salt treatment.

\begin{tabular}{|c|c|c|c|c|}
\hline Gene ID & Function description & Tair ID & $\log _{2} \mathrm{FC}$ & $p$-Value \\
\hline Potri.005G064300 & ABC-2 type transporter family protein & AT2G13610.1 & 1.60 & 0.00020675 \\
\hline Potri.014G081000 & acetyl Co-enzyme a carboxylase carboxyltransferase alpha subunit & AT2G38040.2 & 1.83 & $5.05 \times 10^{-10}$ \\
\hline Potri.005G229700 & ADPGLC-PPase large subunit & AT1G27680.1 & -1.38 & $6.79 \times 10^{-9}$ \\
\hline Potri.001G173700 & alpha/beta-Hydrolases superfamily protein & AT3G15650.2 & -2.80 & $6.58 \times 10^{-8}$ \\
\hline Potri.004G188100 & arogenate dehydratase 6 & AT1G08250.1 & -1.78 & $1.45 \times 10^{-16}$ \\
\hline Potri.002G057700 & ATP-dependent caseinolytic $(\mathrm{Clp})$ protease/crotonase family protein & AT1G06550.1 & -1.02 & $1.36 \times 10^{-6}$ \\
\hline Potri.019G089000 & basic helix-loop-helix (bHLH) DNA-binding superfamily protein & AT1G68810.1 & 3.53 & $2.90 \times 10^{-12}$ \\
\hline Potri.003G167500 & BCL-2-associated athanogene 5 & AT1G12060.1 & -1.67 & $8.68 \times 10^{-5}$ \\
\hline Potri.007G055100 & BTB and TAZ domain protein 4 & AT5G67480.2 & -1.53 & $4.51 \times 10^{-10}$ \\
\hline Potri.008G103900 & Ca2+-binding protein 1 & AT5G49480.1 & -1.38 & $2.76 \times 10^{-5}$ \\
\hline Potri.013G112500 & calcium-dependent protein kinase 2 & AT1G35670.1 & 1.88 & $4.92 \times 10^{-10}$ \\
\hline Potri.008G160200 & CBL-interacting protein kinase 4 & AT4G14580.1 & -1.29 & 0.00029303 \\
\hline Potri.001G066400 & CCR-like & AT3G26740.1 & 2.56 & $1.20 \times 10^{-22}$ \\
\hline Potri.010G113400 & Chaperone DnaJ-domain superfamily protein & AT1G71000.1 & -1.49 & $1.49 \times 10^{-9}$ \\
\hline Potri.010G084300 & CVP2 like 1 & AT2G32010.2 & -2.15 & $4.23 \times 10^{-13}$ \\
\hline Potri.001G351400 & cyclophilin 38 & АT3G01480.1 & 1.13 & $5.07 \times 10^{-5}$ \\
\hline Potri.005G229500 & dihydroflavonol 4-reductase & AT5G42800.1 & -1.38 & $2.02 \times 10^{-10}$ \\
\hline Potri.005G113700 & flavanone 3-hydroxylase & AT3G51240.1 & -1.10 & $1.53 \times 10^{-6}$ \\
\hline Potri.012G106500 & Glycosyl hydrolase family 38 protein & AT5G13980.2 & 1.06 & $3.09 \times 10^{-5}$ \\
\hline Potri.016G138600 & Glycosyl hydrolase superfamily protein & AT5G01930.1 & 1.42 & 0.00014768 \\
\hline Potri.008G151700 & Haloacid dehalogenase-like hydrolase (HAD) superfamily protein & AT2G32150.1 & -1.24 & $1.56 \times 10^{-7}$ \\
\hline Potri.004G073600 & heat shock protein 90.1 & AT5G52640.1 & -1.18 & $2.27 \times 10^{-7}$ \\
\hline Potri.008G165200 & HR-like lesion-inducing protein-related & AT4G14420.1 & -1.33 & $6.27 \times 10^{-7}$ \\
\hline Potri.009G070800 & Lateral root primordium (LRP) protein-related & AT5G12330.4 & 1.27 & 0.00040463 \\
\hline Potri.012G088100 & Leucine-rich receptor-like protein kinase family protein & AT5G56040.2 & 2.70 & 0.00026452 \\
\hline Potri.001G113100 & leucoanthocyanidin dioxygenase & AT4G22880.2 & -1.49 & $1.07 \times 10^{-12}$ \\
\hline Potri.019G067500 & Major facilitator superfamily protein & AT1G59740.1 & 2.81 & 0.00010361 \\
\hline Potri.002G173900 & myb domain protein 3 & АT3G13540.1 & -1.19 & $2.27 \times 10^{-7}$ \\
\hline Potri.006G178700 & NAD(P)-binding Rossmann-fold superfamily protein & AT2G23910.1 & -1.11 & $3.45 \times 10^{-5}$ \\
\hline Potri.008G116500 & NAD(P)-binding Rossmann-fold superfamily protein & AT1G75290.1 & -1.40 & $1.31 \times 10^{-10}$ \\
\hline Potri.002G234000 & NAD(P)-linked oxidoreductase superfamily protein & AT1G60690.1 & 2.08 & $7.28 \times 10^{-5}$ \\
\hline Potri.019G093400 & nine-cis-epoxycarotenoid dioxygenase 4 & AT4G19170.1 & 1.44 & $1.14 \times 10^{-10}$ \\
\hline Potri.008G186500 & Octicosapeptide/Phox/Bem1p family protein & АT3G26510.4 & 1.27 & $8.82 \times 10^{-8}$ \\
\hline Potri.001G404600 & Peroxisomal membrane $22 \mathrm{kDa}(\mathrm{Mpv} 17 / \mathrm{PMP} 22)$ family protein & AT1G52870.2 & 2.27 & $6.27 \times 10^{-19}$ \\
\hline Potri.016G091100 & PHE ammonia lyase 1 & AT2G37040.1 & -1.60 & $6.90 \times 10^{-15}$ \\
\hline Potri.008G186600 & phosphate transporter 2;1 & AT3G26570.2 & 2.17 & $7.95 \times 10^{-8}$ \\
\hline Potri.011G142300 & photosystem II subunit R & AT1G79040.1 & 1.70 & $6.07 \times 10^{-10}$ \\
\hline Potri.006G011200 & Protein of unknown function (DUF594) & AT5G45460.1 & 2.68 & $6.81 \times 10^{-10}$ \\
\hline Potri.010G210000 & PsbP-like protein 2 & AT2G39470.1 & 2.01 & $1.41 \times 10^{-12}$ \\
\hline Potri.001G001600 & Pyruvate kinase family protein & AT5G56350.1 & -1.15 & $1.15 \times 10^{-18}$ \\
\hline Potri.001G098300 & respiratory burst oxidase protein $\mathrm{F}$ & AT1G64060.1 & -1.19 & $5.42 \times 10^{-5}$ \\
\hline Potri.001G055300 & Rubber elongation factor protein (REF) & AT1G67360.2 & -1.07 & $3.27 \times 10^{-19}$ \\
\hline Potri.017G134900 & transmembrane kinase 1 & AT1G66150.1 & 3.77 & $7.90 \times 10^{-11}$ \\
\hline Potri.002G195800 & TRICHOME BIREFRINGENCE-LIKE 6 & АT3G62390.1 & -1.81 & $3.95 \times 10^{-6}$ \\
\hline Potri.006G095000 & tubulin beta 8 & AT5G23860.2 & -1.86 & $2.57 \times 10^{-10}$ \\
\hline Potri.013G118700 & UDP-glucosyl transferase 78D2 & AT5G17050.1 & -1.73 & $1.81 \times 10^{-10}$ \\
\hline Potri.014G039000 & Uncharacterised protein family (UPF0114) & AT4G19390.1 & -2.55 & $1.71 \times 10^{-1}$ \\
\hline Potri.005G064300 & ABC-2 type transporter family protein & AT2G13610.1 & 1.60 & $4.13 \times 10^{-11}$ \\
\hline
\end{tabular}

\section{Discussion}

Salt stress decreases tree growth and productivity by reducing photosynthetic efficiency, as well as through ion toxicity and osmotic stress. Considering the ongoing expansion of salty land, salt-tolerant plants are in high demand, especially in China, where the total area of salty land available is $3.67 \times 107 \mathrm{hm}^{2}$ [5]. Tolerance to salt is complex, with variable effects occurring at both the molecular and physiological levels during different stages of plant development. Salt adaptation mechanisms are normally controlled by multiple genes, and as TFs regulate the expression of several genes related to salt stress defense responses, they have great potential in genetic engineering of trees. Significant evidence has shown that DREB/CBF proteins play crucial roles in regulating plant responses to salt and other abiotic stresses [12]. Overexpression of DREB TFs from Vigna radiata and soybean confers salinity tolerance to Arabidopsis thaliana. In the present study, we studied the role of AhDREB in salt tolerance in Populus.

Photosynthesis is one of the most important and fundamental physiological processes during plant growth, but it can be seriously impacted during salt stress. Salt stress may damage the electron transport system and decrease $\mathrm{CO}_{2}$ availability by limiting Gs or altering photosynthetic 
metabolism [38]. In addition, the accumulation of photosynthetic pigments such as chlorophyll, which captures solar radiation to drive the photosynthetic mechanism, is a potential biochemical indicator of salt tolerance [39]. In our study, salt stress decreased chlorophyll content in both transgenic and non-transgenic lines, and the chlorophyll decrement in the non-transgenic line was significantly greater than those in the transgenic lines under $0.6 \%$ and $0.8 \% \mathrm{NaCl}$ treatments, which may be partly responsible for the higher Pn of transgenic lines under salt stress. Aside from Pn, transgenic lines also exhibited higher Gs and Tr, which may be associated with the capacity for salt tolerance.

MDA levels and electrolyte leakage are well-known indicators of plant cell impairment under salt and other abiotic stresses [40]. In our study, transgenic lines exhibited significantly lower levels of MDA and electrolyte leakage, indicating that these transgenic lines have greater salt tolerance compared to the non-transgenic line.

ROS such as superoxide radicals, hydrogen peroxide and singlet oxygen, which are highly toxic and can damage proteins, lipids, carbohydrates and DNA, may be generated in plants under salt stress [41,42]. To counter the toxicity of ROS, plants produce ROS scavengers. Non-enzymatic antioxidants include ascorbic acid and reduced glutathione, and enzymatic antioxidants include SOD and POD, which can accumulate rapidly under drought conditions to minimize oxidative damage [42-45]. In addition, in high-salt environments, plants can also maintain their water content and osmotic potential by accumulating compatible organic solutes, such as proline [46,47]. Proline is an important osmotic protectant that has been suggested to protect enzymes and membranes, scavenge ROS, and supply energy and $\mathrm{N}$ for utilization under salt stress [48]. In the present study, the transgenic T-46 and T-12 lines showed higher SOD and POD activities and greater proline content under salt stress, indicating that $A h D R E B$ transformation enhanced salt tolerance through stimulation of the antioxidant defense system and proline production in Populus.

Genes containing DRE elements in their promoters are activated by overexpression of DREB TFs, resulting in improved stress tolerance in transgenic plants [25]. Although few studies have investigated the target genes of $A h D R E B$, some research has shown that overexpression of AtDREB1A in transgenic Arabidopsis enhances the expression of target stress-response genes and activates multiple stress response mechanisms [49-51]. Yao et al. [16] also showed that ectopic expression of AtDREB1A in transgenic Salvia miltiorrhiza activated stress-response genes such as kin1 and kin2, and protective proteins like proline-rich protein 4 also increased under stress. Stress-response genes including GmDREB6, GmP5CS and GmERF7 were up-regulated in 35S: OsDREB transgenic rice [52]. In our study, to elucidate the interactions of AhDREB1 with other genes that enhance salt tolerance in Populus, we identified genes that were differentially expressed between the non-transgenic line 401 and transgenic lines T-46 and T-12 under salt stress. We found that some stress-related genes were up-regulated in T-46, such as mitogen-activated protein kinase 3 (MAPK3), which plays an important role in mediating stress responses in eukaryotic organisms. Mao et al. showed that MPK3 and MPK6 phosphorylate the TF WRKY33, which was also up-regulated in our study, thereby triggering the synthesis of camalexin, a major antimicrobial phytoalexin in Arabidopsis. Pitzschkea et al. found that MPK3 could phosphorylate AZI1, a lipid transfer protein (LTP)-related hybrid proline-rich protein (HyPRP), to form a protein complex in plants that alleviated salt stress. We also found a number of ROS-responsive genes (including peroxidase superfamily protein, glutathione peroxidase, heat shock transcription factor, and calmodulin-binding protein) were up-regulated in transgenic poplars suffering from salt stress, indicating that $A h D R E B$ expression may modulate activity in ROS-scavenging pathways.

Moreover, we discovered that a multitude of photosynthesis-related genes were up-regulated in T-46 plants in response to salt, suggesting that $A h D R E B$ maintained photosynthetic capacity through regulation of these genes. Previous research has also shown that overexpression of DREB in host plants promoted the expression of photosynthetic genes under abiotic stress conditions. Numerous signal transduction genes, such as G-protein alpha subunit 1, RAB GTPase homolog A5A and CYCLIN D3, as well as various TFs, such as DREB, zinc finger, WRKY, bZIP TF and MYB, were also up-regulated [53]. These genes are involved in mechanisms of plant defense against abiotic stresses. 
AhDREB may drive a complex signaling network that enhances salt tolerance. Although overexpression of DREB TF could improve salt stress, poor growth phenotypes in DREB transgenic plants compared to non-transgenic controls under normal growing conditions have been observed in many studies. For example, overexpression of MsDREB6.2 in apple resulted in severe growth retardation under normal growing conditions [23]. Similarly, transgenic wheat and barley constitutively overexpressing TaDREB2 or TaDREB3 showed slower growth, delayed flowering and lower grain yields than non-transgenic controls [54], and overexpression of a peach DREB resulted in lower growth and phenological changes in transgenic apple plants [55]. Growth inhibition and phenotypic aberrations may be due to the destruction of normal gibberellin, auxin or cytokinin metabolism by foreign genes [23,56]. On the other hand, Wei et al. overexpressed Arabidopsis DREB1A and DREB1B in transgenic Salvia miltiorrhiza, which increased tolerance to drought stress without stunting growth. Similarly, Chen et al. [57] overexpressed PeDREB2 in transgenic tobacco, which increased salt resistance and did not cause growth retardation, indicating that not all DREB genes lead to growth retardation when overexpressed. Selecting a DREB that can increase tolerance without stunting growth is a potential strategy for transgenic salt-resistant poplar breeding.

In our study, growth retardation was not observed in $A$ hDREB transgenic poplar under non-stress conditions, despite several genes being differentially expressed in transgenic poplar according to the transcriptome results. These results indicated that the transcriptomic changes observed in $A h D R E B$ transgenic poplar may not influence the growth of the receptor plant. This result was similar to that of Jiang et al. [28]. In their study, they identified only 7 and 28 differentially expressed genes under salt stress and non-stress conditions, respectively, in a GmDREB transgenic wheat that showed no significant growth retardation. In contrast, in a study of ABF3 transgenic Arabidopsis, Abdeen et al. [30] found three transgenic lines with only seven, one and eight differentially expressed genes under non-stress conditions, all of which showed markedly decreased growth. These studies indicate that RNA sequencing may not reliably predict the characteristics of transgenic plants. Chan et al. [58] suggested that the extent of global transcriptomic differences between transgenic and non-transgenic plants may not predict the phenotypic differences upon which the environment and selection act to influence fitness and fecundity, but the categorical changes may be used to provide guidance for risk assessment.

In our study, several genes associated with plant or cell growth, such as growth-regulating factor 7, wall-associated kinase 2 and photosystem II reaction center protein, were down-regulated in transgenic plants. Growth-regulating factors are plant-specific TFs that participate in regulation of plant growth and development. Kim et al. found that growth-regulating factor 7 functions as a repressor of multiple osmotic stress-responsive genes, preventing growth inhibition under normal conditions in Arabidopsis. Wall-associated kinases are proteins that bind to pectin molecules of the cell wall, spanning the plasma membrane. Mutation of wall-associated kinase 2 in Arabidopsis led to dependence on sugars and salts for seedling growth. These results suggest that $A h D R E B$ may influence the growth of receptor plants by regulating some growth-associated genes, despite significant growth reduction not being observed in $A h D R E B$ transgenic lines. In addition, cellulose synthase-related genes, such as cellulose synthase family protein and cellulose synthase-like D5, as well as laccase, which is related to lignin synthase, were up-regulated in transgenic poplars, indicating that the cellulose and lignin contents of the wood of transgenic poplars may be affected. Aside from reduced vegetative growth, overexpression of DREB may also influence the flowering time or fruit size in receptor plants. For example, Suo et al. [59] overexpressed the AtDREB1A gene in soybean, which up-regulated the expression of a GmVRN1-like gene in the vernalization pathway, causing delayed flowering. In our study, the results showed that 16 genes associated with pollen development were down-regulated, including S-locus lectin protein kinase family protein, ELF4-like 4 and B-box type zinc finger family protein, indicating that $A h D R E B$ overexpression in poplar may influence its reproductive development. The transcriptomic results indicated that overexpression of $A h D R E B$ in poplar may influence the cellulose and lignin contents, as well as reproductive growth. 


\section{Conclusions}

In conclusion, we showed that heterologous expression of $A h D R E B$ in poplar could improve salt resistance without stunting growth. The observed enhanced salt tolerance may be related to mediation of the expression of genes related to stress resistance. However, development of DREB-transformed transgenic plants with tolerance to salt stress remains in the greenhouse experimental phase, and thus there is little information on the performance of DREB plants under field conditions. In addition, further study is needed into whether $A$ hDREB influences important characteristics such as wood properties. Future studies may provide insight into stress tolerance mechanisms under both greenhouse and field conditions, and could identify other molecular traits and unintended effects associated with AhDREB.

Supplementary Materials: The following are available online at http:/ /www.mdpi.com/1999-4907/10/3/214/s1. Table S1: The average electrical conductivity of substrate; Table S2: Transcriptome comparison statistics; Table S3: The functional analysis of differential expressed genes in transgenic poplar under salty stress.

Author Contributions: Y.L. designed the experiments, Q.G. wrote the paper, Q.G., N.L., Y.S., W.L. and Z.L. performed the experiments and analyzed the data, H.Z., Q.J., Q.Y., S.C. and W.Z. participated in the and help to complete the experiments.

Acknowledgments: The authors appreciate the financial support from the Science and Technology Development Center of the State Forestry Administration Project (JC-2017-02), the National Science and Technology Major Project of the Ministry of Science and Technology of China (2018ZX08020002-003-002), the Fundamental Research Funds for the Central Universities(2016BLPX13) and the National High Technology Research and Development Program of China (2013AA102703).

Conflicts of Interest: The authors declare no conflict of interest.

\section{References}

1. Zhu, Z.T.; Zhang, Z.Y. The status and advances of genetic improvement of Populus tomentosa Carr. J. Beijing For. Univ. 1997, 6, 1-7.

2. Li, J.G.; Pu, L.J.; Han, M.J.; Zhu, M.; Zhang, R.S.; Xiang, Y.Z. Soil salinization research in China: Advances and prospects. J. Geogr. Sci. 2014, 24, 943-960. [CrossRef]

3. Choudhury, F.K.; Rivero, R.M.; Blumwald, E.; Mittler, R. Reactive oxygen species, abiotic stress and stress combination. Plant J. 2017, 90, 856-867. [CrossRef] [PubMed]

4. Hossain, M.A.; Li, Z.G.; Hoque, T.S.; Burritt, D.J.; Fujita, M.; Munné-Bosch, S. Heat or cold priming-induced cross-tolerance to abiotic stresses in plants: key regulators and possible mechanisms. Protoplasma 2017, 1-14. [CrossRef] [PubMed]

5. Ding, M.Q.; Hou, P.C.; Shen, X.; Wang, M.J.; Deng, S.R.; Sun, J.; Xiao, F.; Wang, R.G.; Zhou, X.Y.; Lu, C.F.; et al. Salt-induced expression of genes related to $\mathrm{Na}+/ \mathrm{K}+$ and $\mathrm{ROS}$ homeostasis in leaves of salt-resistant and salt-sensitive poplar species. Plant Mol. Biol. 2010, 73, 251-269. [CrossRef] [PubMed]

6. Hmida-Sayari, A.; Gargouri-Bouzid, R.; Bidani, A.; Jaoua, L.; Savoure, A.; Jaoua, S. Overexpression of delta (1)-pyrroline-5-carboxylate synthetase increases proline production and confers salt tolerance in transgenic potato plants. Plant Sci. 2005, 169, 746-752. [CrossRef]

7. Wang, S.Y.; Chen, Q.J.; Wang, W.L.; Wang, X.C.; Lu, M.Z. Salt tolerance conferred by over-expression of OsNHX1 gene in Poplar 84K. Chin. Sci. Bull. 2005, 50, 225-229. [CrossRef]

8. Shih, M.D.; Hoekstra, F.A.; Hsing, Y.I.C. Late embryogenesis abundant proteins. Adv. Bot. Res. 2008, 48, 211-255. [CrossRef]

9. Kumar, V.; Shriram, V.; Kishor, P.B.K.; Narendra, J.L.; Shitole, M.G. Enhanced proline accumulation and salt stress tolerance of transgenic indica rice by over-expressing P5CSF129A gene. Plant Biotechnol. Rep. 2010, 4 , 37-48. [CrossRef]

10. Lan, T.; Gao, J.; Zeng, Q.Y. Genome-wide analysis of the LEA (late embryogenesis abundant) protein gene family in Populus trichocarpa. Tree Genet. Genomes 2013, 9, 253-264. [CrossRef]

11. Magwanga, R.O.; Lu, P.; Kirungu, J.N.; Lu, H.J.; Wang, X.X.; Cai, X.Y.; Zhou, Z.L.; Zhang, Z.M.; Salih, H.; Wang, K.B.; et al. Characterization of the late embryogenesis abundant (LEA) proteins family and their role in drought stress tolerance in upland cotton. BMC Genet. 2018, 19, 6. [CrossRef] [PubMed] 
12. Hirayama, T.; Shinozaki, K. Research on plant abiotic stress responses in the post-genome era: Past, present and future. Plant J. 2010, 61, 1041-1052. [CrossRef] [PubMed]

13. Jain, D.; Chattopadhyay, D. Role of DREB-like proteins in improving stress tolerance of transgenic crops. In Plant Acclimation to Environmental Stress; Springer: New York, NY, USA, 2013; pp. 147-161. [CrossRef]

14. Hu, L.; Lu, H.; Liu, Q.; Chen, X.M.; Jiang, X.N. Overexpression of mtlD gene in transgenic Populus tomentosa improves salt tolerance through accumulation of mannitol. Tree Physiol. 2005, 25, 1273-1281. [CrossRef] [PubMed]

15. Wang, Y.C.; Qu, G.Z.; Li, H.Y.; Wu, Y.J.; Wang, C.; Liu, G.F.; Yang, C.P. Enhanced salt tolerance of transgenic poplar plants expressing a manganese superoxide dismutase from Tamarix androssowii. Mol. Biol. Rep. 2010, 37, 1119-1124. [CrossRef] [PubMed]

16. Yao, W.; Wang, S.; Zhou, B.; Zhou, B.R.; Jiang, T.B. Transgenic poplar overexpressing the endogenous transcription factor ERF76 gene improves salinity tolerance. Tree Physiol. 2016, 36, 896-908. [CrossRef] [PubMed]

17. Sakuma, Y.; Liu, Q.; Dubouzet, J.G.; Abe, H.; Shinozaki, K.; Yamaguchi-Shinozaki, K. DNA-binding specificity of the ERF/AP2 domain of Arabidopsis DREBs, transcription factors involved in dehydration-and cold-inducible gene expression. Biochem. Biophys. Res. Commun. 2002, 290, 998-1009. [CrossRef] [PubMed]

18. Dubouzet, J.G.; Sakuma, Y.; Ito, Y.; Kasuga, M.; Dubouzet, E.G.; Miura, S.; Seki, M.; Shinozaki, K.; Yamaguchi-Shinozaki, K. OsDREB genes in rice, Oryza sativa L., encode transcription activators that function in drought-, high-salt-and cold-responsive gene expression. Plant J. 2003, 33, 751-763. [CrossRef] [PubMed]

19. Li, X.P.; Tian, A.G.; Luo, G.Z.; Gong, Z.Z.; Zhang, J.S.; Chen, S.Y. Soybean DRE-binding transcription factors that are responsive to abiotic stresses. Theor. Appl. Genet. 2005, 110, 1355-1362. [CrossRef] [PubMed]

20. Liu, S.X.; Wang, X.L.; Wang, H.W.; Xin, H.B.; Yang, X.H.; Yan, J.B.; Li, J.S.; Tran, L.P.; Shinozaki, K.; Yamaguchi-Shinozaki, K.; et al. Genome-wide analysis of ZmDREB genes and their association with natural variation in drought tolerance at seedling stage of Zea mays L. PloS Genet. 2013, 9, e1003790. [CrossRef] [PubMed]

21. Artlip, T.S.; Wisniewski, M.E.; Bassett, C.L.; Norelli, J.L. CBF gene expression in peach leaf and bark tissues is gated by a circadian clock. Tree Physiol. 2013, 33, 866-877. [CrossRef] [PubMed]

22. Wu, H.L.; Lv, H.; Li, L.; Mu, S.H.; Li, X.P.; Gao, J. Genome-wide analysis of the AP2/ERF transcription factors family and the expression patterns of DREB genes in Moso Bamboo (Phyllostachys edulis). PLoS ONE 2015, 10, e0126657. [CrossRef] [PubMed]

23. Liao, X.; Guo, X.; Wang, Q.; Wang, Y.T.; Zhao, D.; Yao, L.P.; Wang, S.; Liu, G.J.; Li, T.H. Overexpression of MsDREB6.2 results in cytokinin-deficient developmental phenotypes and enhances drought tolerance in transgenic apple plants. Plant J. 2017, 89, 510-526. [CrossRef] [PubMed]

24. Atia, A.; Barhoumi, Z.; Debez, A.; Hkiri, S.; Abdelly, C.; Smaoui, A.; Chaffei, C.; Gouia, H. Plant Hormones: Potent Targets for Engineering Salinity Tolerance in Plants. In Salinity Responses and Tolerance in Plants; Springer: New York, NY, USA, 2018; Volume 1, pp. 159-184. [CrossRef]

25. Agarwal, P.K.; Agarwal, P.; Reddy, M.K.; Sopory, S.K. Role of DREB transcription factors in abiotic and biotic stress tolerance in plants. Plant Cell Rep. 2006, 25, 1263-1274. [CrossRef] [PubMed]

26. Nakashima, K.; Ito, Y.; Yamaguchi-Shinozaki, K. Transcriptional regulatory networks in response to abiotic stresses in Arabidopsis and grasses. Plant Physiol. 2018, 149, 88-95. [CrossRef] [PubMed]

27. Jacob, P.; Hirt, H.; Bendahmane, A. The heat-shock protein/chaperone network and multiple stress resistance. Plant Biotechnol. J. 2017, 15, 405-414. [CrossRef] [PubMed]

28. Jiang, Q.Y.; Niu, F.J.; Sun, X.J.; Hu, Z.; Li, X.H.; Ma, Y.Z.; Zhang, H. RNA-seq analysis of unintended effects in transgenic wheat overexpressing the transcription factor GmDREB1. Crop J. 2017, 5, 207-218. [CrossRef]

29. Miki, B.; Abdeen, A.; Manabe, Y.; MacDonald, P. Selectable marker genes and unintended changes to the plant transcriptome. Plant Biotechnol. J. 2009, 7, 211-218. [CrossRef] [PubMed]

30. Abdeen, A.; Schnell, J.; Miki, B. Transcriptome analysis reveals absence of unintended effects in drought-tolerant transgenic plants overexpressing the transcription factor ABF3. BMC Genom. 2010, 11, 69. [CrossRef] [PubMed]

31. Quan, R.D.; Shang, M.; Zhang, H.; Zhao, Y.X.; Zhang, J.R. Improved chilling tolerance by transformation with bet $A$ gene for the enhancement of glycinebetaine synthesis in maize. Plant Sci. 2004, 166, 141-149. [CrossRef] 
32. Bates, L.S.; Waldren, R.P.; Teare, I.D. Rapid determination of free proline for water-stress studies. Plant Soil 1973, 39, 205-207. [CrossRef]

33. Becana, M.; Aparicio-Tejo, P.; Irigoyen, J.J.; Sanchez-Diaz, M. Some enzymes of hydrogen peroxide metabolism in leaves and root nodules of Medicago sativa. Plant Physiol. 1986, 82, 1169-1171. [CrossRef] [PubMed]

34. Civello, P.M.; Martinez, G.A.; Chaves, A.R.; Anon, M.C. Peroxidase from strawberry fruit (Fragaria ananassa Duch.): partial purification and determination of some properties. J. Agric. Food Chem. 1995, 43. [CrossRef]

35. Sun, H.P.; Li, F.; Xu, Z.J.; Sun, M.L.; Cong, H.Q.; Qiao, F.; Zhong, X.H. De novo leaf and root transcriptome analysis to identify putative genes involved in triterpenoid saponins biosynthesis in Hedera helix L. PLOS ONE 2017, 12, e0182243. [CrossRef] [PubMed]

36. Mortazavi, A.; Williams, B.A.; McCue, K.; Schaeffer, L.; Wold, B. Mapping and quantifying mammalian transcriptomes by RNA-Seq. Nat. Methods 2008, 5, 621-628. [CrossRef] [PubMed]

37. Du, Q.; Wang, L.; Yang, X.; Gong, C.; Zhang, D. Populus endo- $\beta$-1,4-glucanases gene family: genomic organization, phylogenetic analysis, expression profiles and association mapping. Planta 2015, 241, 1417-1434. [CrossRef] [PubMed]

38. Moud, A.M.; Maghsoudi, K. Salt stress effects on respiration and growth of germinated seeds of different wheat (Triticum aestivum L.) cultivars. World J. Agric. Sci. 2008, 4, 351-358.

39. Moradi, F.; Ismail, A.M. Responses of photosynthesis, chlorophyll fluorescence and ROS-scavenging systems to salt stress during seedling and reproductive stages in rice. Ann. Bot. 2007, 99, 1161-1173. [CrossRef] [PubMed]

40. Shan, C.; Liu, H.; Zhao, L.; Wang, X. Effects of exogenous hydrogen sulfide on the redox states of ascorbate and glutathione in maize leaves under salt stress. Biol. Plantarum 2014, 58, 169-173. [CrossRef]

41. Ramanjulu, S.; Bartels, D. Drought-and desiccation-induced modulation of gene expression in plants. Plant Cell Environ. 2002, 25, 141-151. [CrossRef] [PubMed]

42. Bao, A.K.; Du, B.Q.; Touil, L.; Kang, P.; Wang, Q.L.; Wang, S.M. Co-expression of tonoplast Cation/H+ antiporter and H+-pyrophosphatase from xerophyte Zygophyllum xanthoxylum improves alfalfa plant growth under salinity, drought and field conditions. Plant Biotechnol. J. 2016, 14, 964-975. [CrossRef] [PubMed]

43. Farooq, M.; Hussain, M.; Wahid, A.; Siddique, K.H.M. Drought stress in plants: An overview. In Plant Responses to Drought Stress; Springer: New York, NY, USA, 2012; pp. 1-33. [CrossRef]

44. Diaz-Vivancos, P.; Barba-Espín, G.; Hernández, J.A. Elucidating hormonal/ROS networks during seed germination: insights and perspectives. Plant Cell Rep. 2013, 32, 1491-1502. [CrossRef] [PubMed]

45. Shi, H.B.; Luo, J.; Yao, D.W.; Zhu, J.J.; Xu, H.F.; Shi, H.P.; Loor, J.J. Peroxisome proliferator-activated receptor- $\gamma$ stimulates the synthesis of monounsaturated fatty acids in dairy goat mammary epithelial cells via the control of stearoyl-coenzyme A desaturase. J. Dairy Sci. 2013, 96, 7844-7853. [CrossRef] [PubMed]

46. Hajiboland, R. Reactive oxygen species and photosynthesis. In Oxidative Damage to Plants; Elsevier: Amsterdam, Netherlands, 2014; pp. 1-63. [CrossRef]

47. Bompy, F.; Lequeue, G.; Imbert, D.; Dulormne, M. Increasing fluctuations of soil salinity affect seedling growth performances and physiology in three Neotropical mangrove species. Plant Soil 2014, 380, 399-413. [CrossRef]

48. Szabados, L.; Savoure, A. Proline: a multifunctional amino acid. Trends Plant Sci. 2010, 15, 89-97. [CrossRef] [PubMed]

49. Kasuga, M.; Liu, Q.; Miura, S.; Yamaguchi-Shinozaki, K.; Shinozaki, K. Improving plant drought, salt, and freezing tolerance by gene transfer of a single stress-inducible transcription factor. Nat. Biotechnol. 1999, 17, 287. [CrossRef] [PubMed]

50. Maruyama, K.; Sakuma, Y.; Kasuga, M.; Ito, Y.; Seki, M.; Goda, H.; Shimada, Y.; Yoshida, S.; Shinozaki, K.; Yamaguchi-Shinozaki, K. Identification of cold-inducible downstream genes of the Arabidopsis DREB1A/ CBF3 transcriptional factor using two microarray systems. Plant J. 2004, 38, 982-993. [CrossRef] [PubMed]

51. Zhao, M.G.; Tian, Q.Y.; Zhang, W.H. Nitric oxide synthase-dependent nitric oxide production is associated with salt tolerance in Arabidopsis. Plant Physiol. 2007, 144, 206-217. [CrossRef] [PubMed]

52. Zhang, X.X.; Tang, Y.J.; Ma, Q.B.; Yang, C.Y.; Mu, Y.H.; Suo, H.C.; Luo, L.H.; Nian, H. OsDREB2A, a rice transcription factor, significantly affects salt tolerance in transgenic soybean. PLoS ONE 2013, 8, e83011. [CrossRef] [PubMed] 
53. Shinozaki, K.; Yamaguchi-Shinozaki, K. Gene expression and signal transduction in water-stress response. Plant Physiol. 1997, 115, 327. [CrossRef] [PubMed]

54. Mondini, L.; Nachit, M.M.; Pagnotta, M.A. Allelic variants in durum wheat (Triticum turgidum L. Szabados var. durum) DREB genes conferring tolerance to abiotic stresses. Mol. Genet. Genom. 2015, 290, 531-544. [CrossRef] [PubMed]

55. Artlip, T.S.; Wisniewski, M.E.; Norelli, J.L. Field evaluation of apple overexpressing a peach CBF gene confirms its effect on cold hardiness, dormancy, and growth. Environ. Exp. Bot. 2014, 106, 79-86. [CrossRef]

56. Li, J.; Sima, W.; Ouyang, B.; Wang, T.; Ziaf, K.; Luo, Z.; Liu, L.; Li, H.; Chen, M.; Huang, Y.; et al. Tomato $S I D R E B$ gene restricts leaf expansion and internode elongation by downregulating key genes for gibberellin biosynthesis. J. Exp. Bot. 2012, 63, 6407-6420. [CrossRef] [PubMed]

57. Chen, J.H.; Xia, X.L.; Yin, W.L. Expression profiling and functional characterization of a DREB2-type gene from Populus euphratica. Biochem. Biophys. Res. Commun. 2009, 378, 483-487. [CrossRef] [PubMed]

58. Chan, Z.; Bigelow, P.J.; Loescher, W.; Grumet, R. Comparison of salt stress resistance genes in transgenic Arabidopsis thaliana indicates that extent of transcriptomic change may not predict secondary phenotypic or fitness effects. Plant Biotechnol. J. 2012, 10, 284-300. [CrossRef] [PubMed]

59. Suo, H.; Ma, Q.; Ye, K.; Yang, C.; Tang, Y.; Hao, J.; Zhang, Z.Y.J.; Nian, H. Overexpression of AtDREB1A causes a severe dwarf phenotype by decreasing endogenous gibberellin levels in soybean [Glycine max (L.) Merr.]. PLoS ONE 2012, 7, e45568. [CrossRef] [PubMed]

(C) 2019 by the authors. Licensee MDPI, Basel, Switzerland. This article is an open access article distributed under the terms and conditions of the Creative Commons Attribution (CC BY) license (http:/ / creativecommons.org/licenses/by/4.0/). 